\title{
Hiperostosis esquelética difusa idiopática, una causa infrecuente de disfagia y disfonía
}

\section{Diffuse idiopathic skeletal hyperostosis, an infrequent cause of dysphagia and hoarseness}

\author{
Noemith Añaguari T. ${ }^{1}$, Susana Arango A. ${ }^{1,2}$, Isabel Zapater S. $^{3}$
}

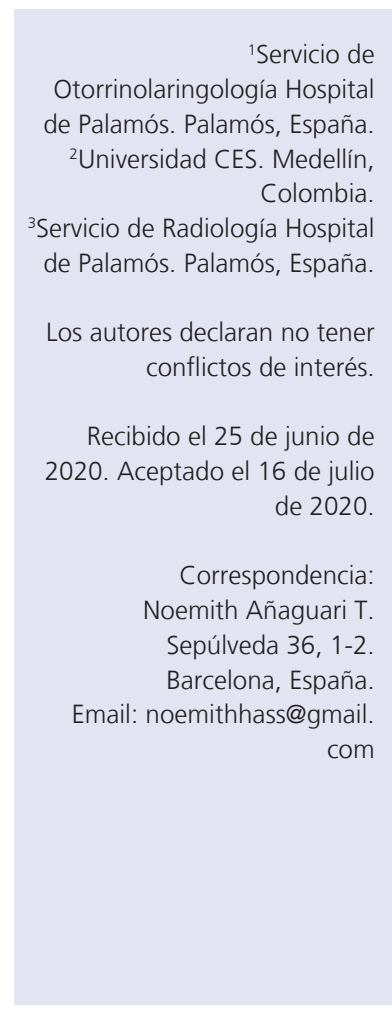

\section{Resumen}

La hiperostosis esquelética difusa idiopática (DISH) es una enfermedad sistémica caracterizada por la osificación del ligamento longitudinal anterior de la columna. Los pacientes suelen ser asintomáticos o presentar dolor leve o rigidez, sin embargo, cuando afecta la región cervical puede ocasionar disfagia, disfonía o disnea. Presentamos el caso de un paciente de 63 años con disfonía y disfagia en quien los estudios demostraron desplazamiento del aritenoides y colapso del seno piriforme debido a un osteofito a nivel de C4. El paciente presentó mejoría con tratamiento conservador. Realizamos una discusión del caso y una revisión de la literatura sobre diagnóstico y tratamiento de esta patología.

Palabras clave: disfonía, disfagia, hiperostosis, osteofito, hiperostosis esquelética difusa idiopática.

\begin{abstract}
Diffuse idiopathic skeletal hyperostosis (DISH) is a systemic disease characterized by ossification of the anterior longitudinal ligament of the spine. Patients are usually asymptomatic, or present mild pain or stiffness, however cervical compromise can cause dysphagia, dyspnea and dysphonia. We present the case of a 63-year-old patient with hoarseness and dysphagia. Studies revealed anterior displacement of the arytenoid cartilage and collapse of the pyriform sinus secondary to an osteophyte at C4 level. The patient showed improvement with conservative management. We present a discussion about this case and the available scientific evidence on the diagnosis and treatment of this pathology.

Keywords: dysphonia, swallowing disorders, hyperostosis, osteophyte, diffuse idiopathic skeletal hyperostosis.
\end{abstract}

\section{Introducción}

La hiperostosis esquelética difusa idiopática DISH por sus siglas en inglés (diffuse idiopathic skeletal hyperostosis) fue descrita por Forestier y Rotes-Querol en $1950^{1}$. La prevalencia de la enfermedad varía entre 2,9\% y $42 \%$ dependiendo de la raza y los criterios diagnósticos utilizados ${ }^{2,3}$. Es una enfermedad sistémica, no inflamatoria, caracterizada por la osificación progresiva de entesis y ligamentos principalmente del esqueleto axial, pero también de las articulaciones periféricas ${ }^{3,4}$. Los síntomas reportados incluyen dolor, rigidez y mayor riesgo de fracturas, aunque la mayoría de pacientes cursan asintomáticos ${ }^{4}$. Las manifestaciones clínicas extraesqueléticas son infrecuentes, la más común es la disfagia que se reporta en 17\%-28\% de los pacientes seguida por el compromiso de la vía aérea y la disfonía $a^{5,6}$. A continuación, presentamos el caso de un paciente que debutó con disfagia a sólidos y disfonía de larga data secundarios a un osteofito a nivel de C4. 


\section{Caso Clínico}

Paciente de sexo masculino de 63 años, con historia de tabaquismo activo de 80 paquetes-año, sin otras patologías crónicas conocidas. Referido por historia de 12 meses de disfagia progresiva a sólidos, con un puntaje de 5 en la escala eating assessment tool-10 (EAT10) y disfonía de predominio vespertino en los últimos 6 meses. Sin historia de pérdida de peso, infecciones respiratorias o aspiraciones. La evaluación de la voz con la escala de GRABS fue 21001. En la nasofibrolaringoscopia se evidenció una protrusión de la pared faríngea posterior derecha, que contacta la epiglotis, con desplazamiento anterior del aritenoides, acortamiento de la cuerda vocal y colapso del seno piriforme ipsilateral, causando asimetría y acumulación de secreciones en el vestíbulo con rebosamiento ocasional (Figura 1).

La movilidad y tensión de las cuerdas estaba conservada al igual que la sensibilidad faríngea. La tomografía computarizada demostró un osteofito de gran tamaño en la región anterior derecha del cuerpo vertebral C4 con compresión del repliegue ariteno-epiglótico y seno piriforme del mismo lado (Figura 2), y osteofitos de menor tamaño desde C5 hasta C7 sugiriendo posible DISH. En la endoscopia digestiva superior no se encontraron hallazgos significativos.

Se indicó tratamiento sintomático con antiinflamatorios no esteroidales (AINEs), logopedia y terapia de la deglución enfocadas a maniobras posturales, modificación de textura y tamaño de alimentos, y ejercicios de tracto vocal semiocluido. Estas se realizaron dos veces por semana durante 2 meses y luego una vez por semana. A los 6 meses se documentó mejoría de la disfagia con disminución de 3 puntos en la escala EAT-10 y persistencia de la disfonía secundaria a vicios fonatorios y fatiga vocal. Ya que la sintomatología residual no afectaba la calidad de vida del paciente se decidió continuar terapia logopédica en casa y vigilancia clínica periódica.

\section{Discusión}

El DISH es una patología degenerativa que ocurre con mayor frecuencia en hombres

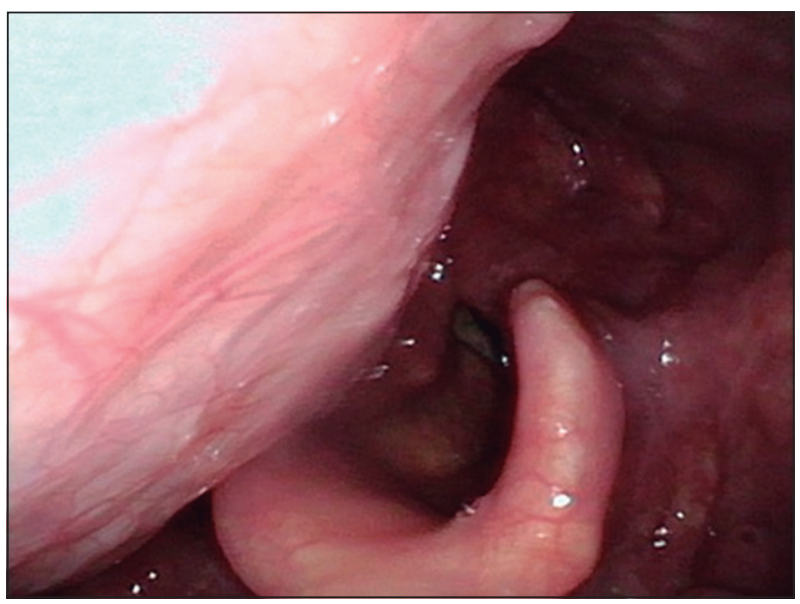

Figura 1. Visión con fibrolaringoscopio flexible. Se observa osteofito a nivel posterior derecho que protruye hacia la luz laríngea, contactando con la epiglotis, produciendo anteversión de aritenoides y colapso del seno piriforme derecho.

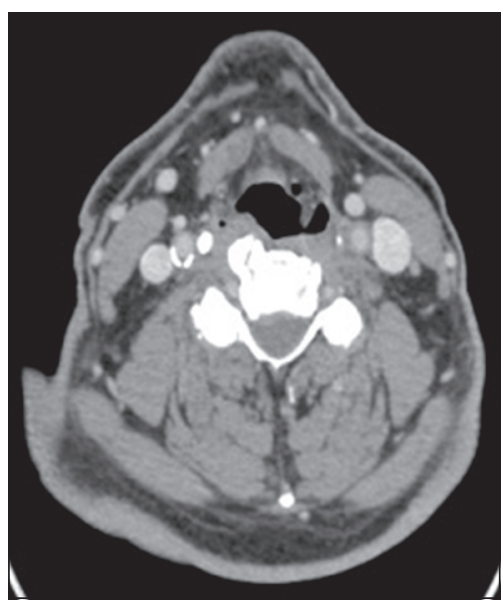

Figura 2. Tomografía computarizada de cueIlo. Corte axial. Se observa osteofito anterior del cuerpo vertebral C4.

que en mujeres y cuya incidencia aumenta progresivamente con la edad ${ }^{7}$. La etiología es desconocida, sin embargo, factores mecánicos y metabólicos como obesidad, hipertensión arterial, diabetes mellitus y dislipidemia se han asociado a su aparición ${ }^{8}$. La región más comúnmente afectada es la torácica, aunque el compromiso cervical ocurre hasta en el $78 \%$ de los casos siendo la mayoría de estos pacientes asintomáticos ${ }^{6}$. La naturaleza y severidad de los síntomas depende de la localización y tamaño de los osteofitos, a nivel cervical pueden ocasionar compresión de las estructuras anatómicas localizadas anteriormente y asociarse 
a disfagia, globus, disnea, estridor, disfonía, apnea del sueño, incluso dificultades para la intubación orotraqueal ${ }^{5-8}$. En 2011, Verlaan y cols. publicaron una revisión sistemática de la literatura donde identificaron 204 pacientes con síntomas aerodigestivos secundarios a DISH, de los cuales $93 \%$ presentaba disfagia, $31 \%$ obstrucción de la vía aérea y 14\% disfonía ${ }^{5}$. La disfagia es de carácter obstructivo, por lo tanto, es más frecuente el compromiso para la deglución de sólidos que líquidos y el empeoramiento con la extensión del cuello 6 . Se clasifica de acuerdo al grado de compromiso con escalas como la propuesta por Miyamoto y cols., quienes la definieron como leve (sensación anormal al deglutir), moderada (dificultad para deglutir sólidos, sin dificultad para líquidos) o severa (inhabilidad para deglutir sólidos y/o tos o aspiración al deglutir $)^{10}$. De igual manera se pueden incluir escalas como el eating assessment tool-10 que permite objetivar la respuesta al tratamiento y predecir el riesgo de aspiración en pacientes con disfagia orofaríngea ${ }^{11}$.

La disfonía puede cuantificarse con la escala GRABS que utiliza parámetros a los cuales se les otorga un puntaje de 0 (normal) a 3 (alteración severa) que se refieren a: G: Grado global de alteración vocal; R: Ronquera; A: Astenia, B: Voz aireada (breathness); S: Espasticidad de la voz (Strain) ${ }^{12}$. En nuestro caso el paciente presentaba disfagia moderada y disfonía leve de predominio vespertino posiblemente secundario a fatiga muscular. Los potenciales mecanismos que dan origen a estos síntomas son: 1) compresión mecánica; 2) edema y fibrosis secundarios a la respuesta inflamatoria de los tejidos adyacentes; 3 ) estrechamiento faríngeo y esofágico por espasmos musculares; 4) neuropatía del nervio laríngeo recurrente por compresión directa e inflamación ${ }^{5,7,9,13}$.

Los criterios diagnósticos más utilizados son los propuestos por Resnick y Niwayama que consisten en: 1) osificación del aspecto anterolateral de al menos cuatro vértebras contiguas; 2) preservación de la altura del disco intervertebral y ausencia de cambios degenerativos y 3 ) ausencia de afectación de las articulaciones sacroilíacas ${ }^{2-5}$. Estos permiten realizar un diagnóstico diferencial con otras patologías similares como espondilitis anquilosante o espondiloartrosis, sin embargo, los criterios propuestos aparecen en etapas tardías de la enfermedad y no abordan su carácter progresivo por lo cual se han propuesto nuevas clasificaciones sin llegar aún a un consenso. La radiografía cervical es útil para el diagnóstico inicial, aunque se recomienda utilizar tomografía ya que permite una mejor caracterización de los osteofitos y el grado de compromiso de estructuras vecinas ${ }^{4}$. Los niveles cervicales que se asocian con mayor grado de disfagia y compromiso de la vía aérea son C4 (78\%), C5 $(63 \%)$ y C3 $(59 \%)^{5}$. En pacientes con sospecha de disfagia obstructiva secundaria a DISH se requiere realizar una videofluoroscopia o una endoscopia digestiva superior para descartar otras causas de compresión íntrinsecas ${ }^{6,9}$.

El tratamiento de la disfagia y disfonía secundaria a DISH debe ser guiado de acuerdo a la severidad de la enfermedad y está dirigido al control de síntomas. El manejo incluye analgésicos, antiinflamatorios no esteroidales (AINEs), relajantes musculares, terapia física y cambios en la dieta ${ }^{4,14}$. El tratamiento quirúrgico se basa en la escisión de los osteofitos a través del abordaje anterolateral y se reserva para casos refractarios al manejo inicial o con compromiso severo e incapacitante. De acuerdo a lo reportado por Verlaan y cols. las complicaciones mayores son infrecuentes (menores al $1 \%$ ) e incluyen empeoramiento de la disfagia o disfonía, requerimiento de traqueotomía en el postoperatorio, lesión nerviosa, accidente cerebrovascular y muerte ${ }^{5,7,10}$. Se ha descrito la recurrencia de nuevos osteofitos, sin embargo, la mayoría de pacientes permanecen asintomáticos ${ }^{7,10,15}$. Nuestro paciente presentó mejoría de la disfagia con tratamiento conservador, la disfonía persistió, pero ante la ausencia de compromiso de la vía aérea se consideró continuar manejo sintomático.

\section{Conclusión}

La hiperostosis esquelética difusa idiopática puede causar disfagia y rara vez ocasiona disfonía, es más frecuente en hombres y aumenta progresivamente con la edad. El diagnóstico se confirma con estudios radiológicos y en caso de compromiso aerodigestivo es necesario realizar endoscopia digestiva o videofluoroscopia para descartar otras causas de compresión. El 
tratamiento definitivo es la escisión quirúrgica de los osteofitos, sin embargo, el manejo conservador tiene resultados satisfactorios en pacientes con síntomas leves a moderados.

\section{Bibliografía}

1. Forestier J, Rotes-Querol J. Senile ankylosing hyperostosis of the spine. Ann Rheum Dis. 1950;9(4):321-30. doi: 10.1136/ard.9.4.321.

2. Kuperus JS, de Gendt EEA, Oner FC, de Jong PA, Buckens SCFM, van der Merwe AE, Maat GJR, Regan EA, Resnick DL, Mader R, Verlaan JJ. Classification criteria for diffuse idiopathic skeletal hyperostosis: a lack of consensus. Rheumatology (Oxford). 2017;56(7):1123-1134. doi: 10.1093/rheumatology/ kex056.

3. Nascimento FA, Gatto LA, Lages RO, Neto HM, Demartini Z, Koppe GL. Diffuse idiopathic skeletal hyperostosis: A review. Surg Neurol Int. 2014;5(Suppl 3):S122-5. doi: 10.4103/2152-7806.130675.

4. Mader R, Verlaan JJ, Eshed I, Bruges-Armas J, Puttini PS, Atzeni F, Buskila D, Reinshtein E, Novofastovski I, Fawaz A, Kurt V, Baraliakos X. Diffuse idiopathic skeletal hyperostosis (DISH): where we are now and where to go next. RMD Open. 2017;3(1):e000472. doi: 10.1136/rmdopen-2017-000472.

5. Verlaan JJ, Boswijk PF, de Ru JA, Dhert WJ, Oner FC. Diffuse idiopathic skeletal hyperostosis of the cervical spine: an underestimated cause of dysphagia and airway obstruction. Spine J. 2011;11(11):1058-67. doi: 10.1016/j.spinee.2011.09.014.

6. Resnick D, Shapiro RF, Wiesner KB, Niwayama G, Utsinger PD, Shaul SR. Diffuse idiopathic skeletal hyperostosis (DISH) [ankylosing hyperostosis of Forestier and Rotes-Querol]. Semin Arthritis Rheum. 1978;7(3):153-187. doi:10.1016/0049-0172(78)900367.

7. Rahimizadeh A, Soufiani H, Rahimizadeh S, Amirzadeh M. Two cases report of dysphagia due to diffuse idiopathic skeletal hyperostosis (DISH). Orthop Res Traumatol Open J. 2018;3(1):26-32. doi: 10.17140/ORTOJ-3-113.

8. Mader R, Verlaan JJ, Buskila D. Diffuse idiopathic skeletal hyperostosis: clinical features and pathogenic mechanisms. Nat Rev Rheumatol. 2013;9(12):741-50. doi: 10.1038/nrrheum.2013.165.

9. Lee JH, Paeng SH, Pyo SY, Kim ST, Lee WH. Swallowing Difficulty in Diffuse Idiopathic Skeletal Hyperostosis with Metabolic Syndrome. Korean J Neurotrauma. 2020;16(1):90-98. doi:10.13004/ kjnt.2020.16.e4.

10. Miyamoto K, Sugiyama S, Hosoe H, Iinuma N, Suzuki Y, Shimizu K. Postsurgical recurrence of osteophytes causing dysphagia in patients with diffuse idiopathic skeletal hyperostosis. Eur Spine J. 2009;18(11):1652-8. doi:10.1007/s00586-009-1133-3.

11. Cheney DM, Siddiqui MT, Litts JK, Kuhn MA, Belafsky PC. The Ability of the 10-Item Eating Assessment Tool (EAT-10) to Predict Aspiration Risk in Persons With Dysphagia. Ann Otol Rhinol Laryngol. 2015;124(5):351-4. doi:10.1177/0003489414558107.

12. Sáenz-Lechón N, Godino-Llorente JI, Osma-Ruiz V, Blanco-Velasco M, Cruz-Roldán F. Automatic assessment of voice quality according to the GRBAS scale. Conf Proc IEEE Eng Med Biol Soc. 2006;2006:2478-81. doi:10.1109/IEMBS.2006.260603.

13. Pulcherio JO, Velasco CM, Machado RS, Souza $\mathrm{WN}$, Menezes DR. Forestier's disease and its implications in otolaryngology: literature review. Braz J Otorhinolaryngol. 2014;80(2):161-6. doi: 10.5935/1808-8694.20140033.

14. Clark E, Preston P, Wates A, Merry P. DISHphagia--a difficult problem to swallow. Rheumatology (Oxford). 2003;42(11):1422-3. doi: 10.1093/rheumatology/ keg353.

15. Mattioli F, Ghirelli M, Trebbi M, Silvestri M, Presutti L, Fermi M. Improvement of Swallowing Function After Surgical Treatment of Diffuse Idiopathic Skeletal Hyperostosis: Our Experience. World Neurosurg. 2020;134:e29-e36. doi: 10.1016/j. wneu.2019.08.124. 